\title{
Inhaltsverzeichnis Band 1: \\ Grundlagen, Systemaufbau und Methoden
}

Inhaltsverzeichnis Band 2:

Betriebsmittel und ihre quasistationäre Modellierung $-X$

Inhaltsverzeichnis Band 3:

Systemverhalten und Berechnung von Drehstromsystemen $-\mathrm{XV}$

Größenbezeichnungen $-\mathrm{XXI}$

1 Einführung und Übersicht -1

2 Stationäre und quasistationäre Zustände des Elektroenergiesystems - 3

3 Komplexe Zeitzeigerdarstellung - 5

3.1 Zusammenhang zeitabhängige Größe und

rotierender Amplitudenzeitzeiger $\mathbf{- 5}$

$3.2 \quad$ Zeitzeiger -6

3.3 Spezielle Zeiger und Versoren -9

3.4 Zeigerdrehungen mit a und $\mathrm{j}-\mathbf{1 0}$

3.5 Spezielle Werte der Winkelfunktionen - 10

3.6 Umrechnungsformeln von Winkelfunktionen - 11

$4 \quad$ Matrizen und Vektoren -13

4.1 Matrizenschreibweise von Gleichungssystemen - 13

4.2 Lösung von linearen Gleichungssystemen - 13

4.3 Spezielle Matrizen - 17

4.4 Rechenregeln für Matrizen - 17

4.5 Koordinatentransformation, Eigenwerte und Eigenvektoren - 19

4.6 Eigenwerte und inverse Matrizen spezieller Matrizen -20

$5 \quad$ Verbraucherzählpfeilsystem, Impedanz und Admittanz — 23

5.1 Zählpfeilzuordnung im Verbraucherzählpfeilsystem — 23

5.2 Impedanz und Admittanz - 24

5.3 Zeigerbilder für typische Grundschaltelemente von Zweipolen im VZS - 26

5.4 Hinweise zur Konstruktion von Zeigerbildern -27

6 Leistungsberechnung und Oberschwingungen - 29

$6.1 \quad$ Leistung im VZS 29 
6.2 Eigenschaften von typischen Grundschaltelementen von Zweipolen im VZS - 31

6.3 Oberschwingungen -33

6.4 Verschiebungsfaktor, Leistungsfaktor und Verzerrungsleistung - 34

$7 \quad$ Zwei-, Vier- und Mehrpoldarstellung - 37

7.1 Satz von der Ersatzspannungsquelle - $\mathbf{3 7}$

7.2 Satz von der Ersatzstromquelle - 38

7.3 Umwandlung Spannungsquellenersatzschaltung in Stromquellenersatzschaltung - 38

7.4 Vier- und Mehrpolgleichungen - 39

7.4.1 Impedanzdarstellung - 39

7.4.2 Admittanzdarstellung $-\mathbf{4 0}$

7.4.3 Kettenformdarstellung - $\mathbf{4 0}$

7.4.4 Spezielle Vierpole und ihre Ersatzschaltungen - 42

8 Kirchhoff'sche Gesetze und Strom- und Spannungsteilerregeln — 45

8.1 Graphen und Subgraphen - 45

8.2 Knotenpunktsatz (1. Kirchhoff'sches Gesetz) - 46

8.3 Maschensatz (2. Kirchhoff'sches Gesetz) - 49

8.4 Topologische Regeln und Anzahl der Gleichungen und Unbekannten - $\mathbf{5 0}$

8.5 Spannungsteilerregel -51

8.6 Stromteilerregel $-\mathbf{5 2}$

9 Drehstromsystem -55

9.1 Vom Wechselstromsystem zum Drehstromsystem - 55

9.2 Stern- und Dreieckschaltung - $\mathbf{5 7}$

9.2.1 Sternschaltung $-\mathbf{5 8}$

9.2.2 Dreieckschaltung - $\mathbf{5 8}$

9.2.3 Bezeichnungen für Spannungen und Ströme - $\mathbf{5 9}$

9.2.4 Zusammenhänge zwischen Außenleiter- und Stranggrößen - 59

9.3 Umrechnungen zwischen Dreieckschaltung und Sternschaltung — 61

9.4 Induktive und kapazitive Kopplung - 62

9.5 Leistung im Drehstromsystem - 63

10 Positionswinkel, Winkelgeschwindigkeit und Drehimpulssatz -65

$10.1 \quad$ Mechanischer und elektrischer Winkel und Winkelgeschwindigkeiten - 65

10.2 Drehimpulssatz -66 
11 Induzierte Spannungen und verkettete Wicklungen - 69

$11.1 \quad$ Induktionsgesetz -69

11.2 Verkettete Wicklungen — 73

11.3 Kraftwirkung auf stromdurchflossene Leiter im Magnetfeld — 74

11.4 Drehmoment einer stromdurchflossenen Leiterschleife im Magnetfeld -76

12 Wärme, Wärmeübertragung und Wärmespeicherung — 77

$12.1 \quad$ Wärmeleitung - 77

$12.2 \quad$ Konvektion -77

12.3 Wärmestrahlung - 78

12.4 Energieerhaltung: 1. Hauptsatz der Thermodynamik -79

$12.5 \quad$ Wärmewiderstand - 79

12.6 Analogie zwischen thermischen und elektrischen Größen - $\mathbf{8 0}$

13 Energiewandlungskette und Elektroenergie - 81

13.1 Energieumwandlungskette - $\mathbf{8 1}$

13.2 Sankey-Diagramm der Energiewandlung - 82

13.3 Bereitstellung der Elektroenergie und Elektrizitätsflussbild - $\mathbf{8 4}$

13.4 Grundbegriffe der Energiewirtschaft — $\mathbf{8 5}$

14 Verläufe und Kenngrößen für Erzeugung und Verbrauch — 87

14.1 (Leistungs-)Ganglinien und (Leistungs-)Dauerlinien - 87

14.2 Kenngrößen zur Charakterisierung der Ganglinien und Dauerlinien - $\mathbf{8 8}$

14.3 Belastungsgrad und Benutzungsstundendauer $-\mathbf{8 8}$

14.4 Ausnutzungsgrad und Ausnutzungsstundendauer - $\mathbf{9 0}$

14.5 Verlustarbeit und Arbeitsverlustfaktor - $\mathbf{9 0}$

14.6 Gleichzeitigkeitsfaktor - 91

14.7 Netzanschlussebenen -93

15 Aufbau von Elektroenergiesystemen - 95

$15.1 \quad$ Wechsel-, Drehstrom- und Gleichstromsysteme - 95

15.2 Aufbau des Drehstromsystems - 96

15.3 Übertragungsnetz -97

$15.4 \quad$ Verteilungsnetz $-\mathbf{1 0 0}$

16 Gestaltung und Planung von Netzen - 105

16.1 HöS- und HS-Netzformen - 106

16.2 MS-Netzformen -107

16.2.1 MS-Strahlennetz - 108

16.2.2 MS-Ringnetz 109

16.2.3 Netze mit Gegenstation — 111 
16.2.4 Stützpunktnetze - 111

16.2.5 Strangnetz -112

16.2.6 MS-Maschennetze - 113

16.3 NS-Netzformen - 113

16.3.1 NS-Strahlennetze - 114

16.3.2 NS-Ringnetze - 115

16.3.3 NS-Maschennetze - $\mathbf{1 1 6}$

16.4 Eigenschaften der Netzformen - 118

16.5 Spannungshaltung und Spannungsregelung mit Transformatoren - $\mathbf{1 1 9}$

$16.6 \quad(n-1)$-Sicherheit - 121

16.7 Versorgungszuverlässigkeit 123

17 Schalter, Sicherungen und Messwandler - 129

$17.1 \quad$ Leistungsschalter - 129

17.2 Lastschalter und Lasttrennschalter - 131

17.3 Trennschalter - 133

$17.4 \quad$ Niederspannungs-Hochleistungs-Sicherungen - 137

17.5 Hochspannungs-Hochleistungs-Sicherungen - 139

17.6 Stoßkurzschlussstrombegrenzer $\left(/_{\mathrm{S}}\right.$-Begrenzer $)-\mathbf{1 4 0}$

17.7 Messwandler - $\mathbf{1 4 1}$

17.7.1 Stromwandler - 142

17.7.2 Spannungswandler -146

17.7.3 Kombinierte Wandler — 149

18 Schaltanlagen und Umspannanlagen - 151

18.1 Übersicht - 151

18.2 Sammelschienensystem - 153

18.3 Prinzipieller Aufbau von Schaltfeldern — 155

18.3.1 HöS- und HS-Schaltfeld - 155

18.3.2 MS-Abzweig - 156

18.3.3 NS-Abzweig - 157

18.4 Sammelschienenschaltungen in Schaltanlagen - 158

18.4.1 Längs- und Querkupplung und Längs- und Quertrennung - 159

18.4.2 Schaltanlagen mit Einfachsammelschienen mit und ohne Längskupplung — 161

18.4.3 Schaltanlagen mit Mehrfachsammelschienen — 161

18.4.4 Schaltanlagen mit Umgehungssammelschienen — 163

18.4.5 Schaltanlage mit 1/2-Leistungsschalter — 164

18.4.6 Schaltanlagen mit Ringsammelschienen - 164

18.5 Schaltungen in Umspannanlagen - 165

18.5.1 HöS/HS-Umspannwerk — 165 
18.5.2 HS/MS-Umspannstation - 166

18.5.3 MS/NS-Netzstation - 169

18.6 Bauweisen von Schaltanlagen und Umspannanlagen - 171

18.6.1 Luftisolierte Freiluftschaltanlagen - $\mathbf{1 7 1}$

18.6.2 Luftisolierte 110-kV-Innenraumschaltanlagen - 176

18.6.3 MS-Inneraumschaltanlagen in Zellenbauweise - 176

18.7 $\quad \mathrm{SF}_{6}-$ Schaltanlagen $-\mathbf{1 7 8}$

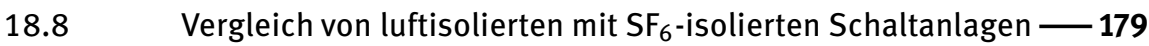

19 Symmetrisches Drehstromsystem und Strangersatzschaltung - 181

19.1 Symmetriebedingungen -183

19.1.1 Elektrische Symmetrie - $\mathbf{1 8 3}$

19.1.2 Geometrische Symmetrie - $\mathbf{1 8 4}$

19.2 Symmetrisches Drehstromsystem — 184

19.3 Strangersatzschaltung - $\mathbf{1 8 5}$

19.4 Dreileiterleistung - $\mathbf{1 8 7}$

19.5 Rechnen mit bezogenen Größen — 187

20 Unsymmetrisches Drehstromsystem und Symmetrische Komponenten - 191

20.1 Ursachen für Unsymmetrie - 191

20.2 Transformation der Leitergrößen in modale Größen

(Modaltransformation) -191

20.3 Leistung in modalen Komponenten — 193

20.4 Symmetrische Komponenten — 193

20.5 Ersatzschaltungen der Symmetrischen Komponenten — 197

20.6 Leistung in Symmetrischen Koordinaten — 199

20.7 Symmetrische Komponenten für spezielle Unsymmetriefälle — 199

$20.8 \quad$ Messung der Mit-, Gegen- und Nullsystemimpedanzen — 202

20.8.1 Mitsystemimpedanz -202

20.8.2 Gegensystemimpedanz -202

20.8.3 Nullsystemimpedanz -203

20.9 Oberschwingungssysteme -204

A Anhang - 207

A.1 Ausgewählte SI-Basis-Einheiten — 207

A.2 Ausgewählte abgeleitete SI-Einheiten — 207

A.3 Naturkonstanten und mathematische Konstanten - 208

Literaturverzeichnis -209

Stichwortverzeichnis -211 